\title{
Diversity-Indicator Based Multi-Objective Evolutionary Algorithm: DI-MOEA*
}

\author{
Yali Wang, Michael T.M. Emmerich, André Deutz, and Thomas Bäck \\ Leiden Institute of Advanced Computer Science, Leiden University, Niels Bohrweg 1, \\ 2333CA Leiden, The Netherlands \\ \{y.wang,m.t.m.emmerich\}@liacs.leidenuniv.nl \\ http://moda.liacs.nl
}

\begin{abstract}
In this paper we propose a Diversity-Indicator based MultiObjective Evolutionary Algorithm (DI-MOEA) for fast computation of evenly spread Pareto front approximations. Indicator-based optimization has been a successful principle for multi-objective evolutionary optimization algorithm (MOEA) design. The idea is to guide the search for approximating the Pareto front by a performance indicator. Ideally, the indicator captures both convergence to the Pareto front and a high diversity, and it does not require a priori knowledge of the Pareto front shape and location. It is, however, so far difficult to define indicators that scale well in computation time for high dimensional objective spaces, and that distribute points evenly on the Pareto front. Moreover, the behavior of commonly applied indicators depends on additional information, such as reference points or sets. The proposed DI-MOEA adopts a hybrid search scheme for combining the advantages of Pareto dominance-based approaches to ensure fast convergence to the Pareto front, with indicator based approaches to ensure convergence to an evenly distributed, diverse set. In addition, it avoids the use of complex structure and parameters in decomposition-based approaches. The Euclidean distance-based geometric mean gap is used as diversity indicator. Experimental results show that the new algorithm can find uniformly spaced Pareto fronts without the involvement of any reference points or sets. Most importantly, our algorithm performs well on both the hypervolume indicator and IGD when comparing with state-of-the-art MOEAs (NSGA-II, SMS-EMOA, MOEA/D and NSGA-III).
\end{abstract}

Keywords: multi-objective optimization $\cdot$ diversity indicator $\cdot$ evolutionary algorithm $\cdot$ indicator-based MOEAs

\section{Introduction}

Many real-world problems require multiple objectives to be optimized, leading us to the so-called "Multi-objective Optimization Problems (MOPs)"[7]. It is

\footnotetext{
* This work is part of the research programme Smart Industry SI2016 with project name CIMPLO and project number 15465, which is (partly) financed by the Netherlands Organisation for Scientific Research (NWO).
} 
usually difficult to find the optimal solutions for MOPs because their objectives are often conflicting with each other, and we are searching for a representative set of Pareto optimal solutions rather than for a single globally optimal solution because no single solution exists that can simultaneously optimize all objectives.

Classical Pareto dominance-based MOEAs, such as NSGA-II [2], use Pareto dominance as a first ranking criterion and use a second ranking criterion to maintain and increase diversity. Pareto dominance-based MOEAs have been a mainstream class for a long time in the field of evolutionary multi-objective optimization (EMO). They are very efficient on MOPs with two or three objectives. However, their performance degrades significantly on many-objective optimization problems (MaOPs), in which the number of objectives is greater than three, due to their ineffectiveness in distinguishing the quality of solutions when the number of objectives becomes large.

As the performance assessment of MOEAs reached a mature stage, performance measures (indicators) on the quality of Pareto front approximations were adopted to search for solutions. These indicators capture both convergence and diversity in a single value. Additionally for Pareto-compliant indicators, it can be shown that they obtain their maximum in a diversified set of solutions on the Pareto front. In general, Indicator-based Evolutionary Algorithms (IBEAs) [14] have strong theoretical support. However, the commonly used performance indicators lead to a convergence in distribution with a high density on the boundary of the Pareto front, as well as on knee regions [1]. If the aim is to obtain uniformly distributed and evenly spread solution sets, so far only indicators that employ an estimate of the true Pareto front as a reference set could be used.

Decomposition $[13,6]$ is a search paradigm that was originally applied by EMO two decades ago [7] and recently regained prominence from the MOEA/D framework [13] and NSGA-III [3]. Decomposition-based MOEAs transform the original multi-objective problem into simpler, single-objective subproblems by means of scalarizations with different weights, therefore they can converge to a well defined, diverse set. However, the central issue in decomposition-based methods is how to select a set of weighting vectors that can provide a well distributed set of Pareto optimal points, given that the location and shape of the Pareto front are unknown a priori. Moreover, the number of weights required to sample a Pareto front with a sufficient resolution suffers a exponential growth from the objective space dimension [6].

Our paper suggests algorithms that combine principles from Pareto dominancebased approach and from indicator-based algorithms. Instead of requiring the indicator to take into account diversity and Pareto dominance, we propose to

- use dominance rank as a primary selection indicator, in order to ensure convergence to the Pareto front;

- use performance indicators that measure the diversity of a set of mutually non-dominated solutions.

However, as opposed to Pareto dominance-based approaches such as SPEA2 and NSGA-II that also maintain diversity, we decide the diversity of a set is 
measured by a scalar value, such that convergence to a maximum diverse set can be achieved and theoretically assessed.

The proposed diversity-indicator based MOEA (DI-MOEA) therefore takes advantage of Pareto dominance-based approaches, and excludes the complex structure and parameters in decomposition-based and contemporary indicatorbased approaches. Most importantly, experimental results show that our algorithm can find well converged and evenly spaced Pareto front approximations without the involvement of any reference points and assumptions about the location and shape of the Pareto front.

The rest of this paper is organized as follows: First, in Section 2, we introduce the diversity indicator. Then, we describe the proposed algorithm in Section 3. Section 4 shows experimental results on benchmark problems. Section 5 concludes the work and outlines some possible future work.

\section{Diversity Indicators and Gap Contribution}

There exist many indicators that assess the diversity of a distribution of points in $\mathbb{R}^{m}$. Among these, the Weitzman indicator and discrepancy measures have excellent theoretical properties, but their computation is expensive. The Hausdorff distance and related measures are indicators that would require the knowledge of the set on which points should be distributed, which is typically not available in Pareto optimization. The Solow-Polasky indicator has been suggested in the context of diversity assessment due to its moderate computational effort and good theoretical properties [10]. However, it is sensitive to the choice of the correlation strength parameter of an exponential kernel function and it requires matrix inversion which might cause numerical instability. The gap indicators (or the averages of distances to nearest neighbours) have been suggested in [4]. They are very fast to compute and easy to implement diversity indicators. In addition, they have certain favorable theoretical properties and empirical results show that their maximization results in diversified, evenly spread approximation sets. These results were obtained for multimodal optimization [12] and evolutionary level set approximation [9] for a wide range of test problems.

Let $A$ define a set of points in $\mathbb{R}^{m}, D(x, A \backslash\{x\})=\min _{a \in A \backslash\{x\}}\{d(x, a)\}$ and $d$ denote the Euclidean distance, then the gap indicators (GI) are defined as follows:

$$
\begin{array}{ll}
G I_{\min }(A)=\min _{x \in A}\{D(x, A \backslash\{x\})\} & \text { Minimal gap } \\
G I_{\Sigma}(A)=\frac{1}{|A|} \sum_{x \in A} D(x, A \backslash\{x\}) & \text { Arithmetic mean gap } \\
G I_{\Pi}(A)=\left(\prod_{x \in A} D(x, A \backslash\{x\})\right)^{\frac{1}{|A|}} & \text { Geometric mean gap }
\end{array}
$$

Note, that $G I_{\min }$ is the well known diversity indicator used in the max-min diversity problem [5]. One can leave out the exponent in $G I_{\Pi}$ and this yields the product distance to the nearest neighbour (PDNN) indicator, considered by Wessing [12] in the context of multimodal optimization. Wessing [12] pointed out 
that $G I_{\Pi \Pi}$ obtains the value of zero in case of duplicates in the set, a property that also holds for $G I_{\min }$. Besides, it can only be used for comparing sets of equal size. Since we are using the indicator contribution as a relative measure of performance of points, these two properties do not cause problems.

In indicator-based steady state selection [1] is to optimize a quality indicator $Q I$ for a solution set. W.l.o.g. we assume the quality indicator is to be maximized. The selection strategy is to add a non-dominated solution $x$ to an approximation set $A$ of size $\mu$ and then retain the best subset $S \subset P$ with $|S|=\mu$ of the new set $P=A \cup\{x\}$. This can be achieved by removing the point that contributes the least to the quality indicator. The indicator contribution of a point $p \in P$ is defined as:

$$
\Delta_{Q I}(p, P) \leftarrow Q I(P)-Q I(P \backslash\{p\})
$$

In our algorithm, the set-indicator contribution of the individual $p \in P$ is defined as the difference of the geometric mean gap indicator value of the set with the individual $p$ minus the indicator-value of the set without it. The computation of the minimal contributor in case of the gap indicators can be solved by computing the solution to the all point nearest neighbour problem (APNN). The straightforward implementation, i. e. measuring distance between all pairs, requires a running time of $O\left(n^{2}\right)$. The APNN problem can be solved by Vaidya's algorithm [11] in optimal time $O(n \log n)$ for a fixed dimensional space and any Minkowski metric, including the Euclidean metric. We propose to choose the Euclidean distance due to its rotational invariance.

\section{Proposed Algorithm}

In the algorithm, we utilize a hybrid selection scheme: the $(\mu+\mu)$ generational selection operator and the $(\mu+1)$ steady state selection operator. The algorithm consists of two components:

- The $(\mu+\mu)$ generational selection operator: When the population is layered to multiple (more than one) dominance ranks, it indicates that the population has not yet converged to the true Pareto front. In this case, the $(\mu+\mu)$ generational selection operator is used to explore the decision space for dominating solutions. In this stage, a strict consideration of the diversity indicator is not yet the key determinant factor. Rather the first priority should be to push the population quickly to the Pareto front. Still, diversity is considered as a secondary ranking criterion in order to bring the points in a good starting position for searching for a uniformly distributed population. Overall, the selection operator is using non-dominated sorting as a primary ranking criterion, then if more than $\mu$ solutions are obtained by adding a layer, we propose two alternative strategies to truncate: the crowding distance (variant 1) as in NSGA-II, and the diversity indicator contribution (variant 2), where points are successively removed in a greedy manner and the contributions are recomputed after each removal. Under the condition that the $\mu$ selected solutions are mutually non-dominated after an iteration, the algorithm switches to the $(\mu+1)$ steady state selection operator. 
- The $(\mu+1)$ steady state selection operator: When the parent population consists of only one non-dominated set, it is likely that the population has already reached a region near the Pareto front. In this case, the indicatorbased $(\mu+1)$ steady state selection operator is applied, as described in Section 2. It discards the least contributor to the quality indicator, here, the diversity indicator. The intent is to achieve a uniformly distributed set on the Pareto front, that is to converge to a maximum of the diversity indicator. If there are more than one dominance ranks in the resulting population, the algorithm switches back to a $(\mu+\mu)$ generational selection operator.

Besides the hybrid selection scheme, another important design choice is the quality indicator, to be specific, the Euclidean distance based geometric mean gap indicator is used to guide the search towards the uniformly distributed Pareto front approximations regardless of the shape of the Pareto front.

The proposed algorithm is presented as pseudo-code in Algorithm 1 and a MOEA-Framework implementation is made available on http://moda.liacs.nl.

\section{Experimental Results and Discussion}

\subsection{Experimental Setup}

In this section, simulations are conducted to demonstrate the performance of the proposed algorithm. Because two different diversity measures are employed in the $(\mu+\mu)$ generational selection operator, two variants of DI-MOEA are involved in the experiments: the crowding distance and the set-indicator contribution are chosen as the second measure in the generational $(\mu+\mu)$ selection operator in algorithm DI-1 and algorithm DI-2 respectively.

All experiments are implemented based on the MOEA Framework 2.1 (http://www.moeaframework.org/), which is a Java-based framework for multiobjective optimization. In the simulations, we use the SBX operator with an index of 15 (30 in NSGA-III and a differential evolution operator is used in MOEA/D.) and polynomial mutation with an index 20. The crossover and mutation probabilities are set to 1 and $1 / N$ respectively and $N$ is the number of variables. In NSGA-III, the number of subdivisions is 99 for bi-objective problems, and 12 for three objective problems. The number of evaluation (NE) is chosen to be dependent on the complexity of the test problem. $20000 \mathrm{NE}$ is used for ZDT problems and $100000 \mathrm{NE}$ for DTLZ problems. The population size is 100 for all problems.

\subsection{Experiments on bi-objective problems}

For bi-objective problems, algorithms are tested on ZDT1, ZDT2 and ZDT3 with 30 variables. Two new algorithms, DI-1 and DI-2, are compared with NSGA-II, SMS-EMOA, NSGA-III and MOEA/D. Table 1 and Table 2 show the aggregate hypervolume and aggregate inverted generational distance (IGD) across 30 runs. The aggregate value is the value obtained when the Pareto solutions from all runs 


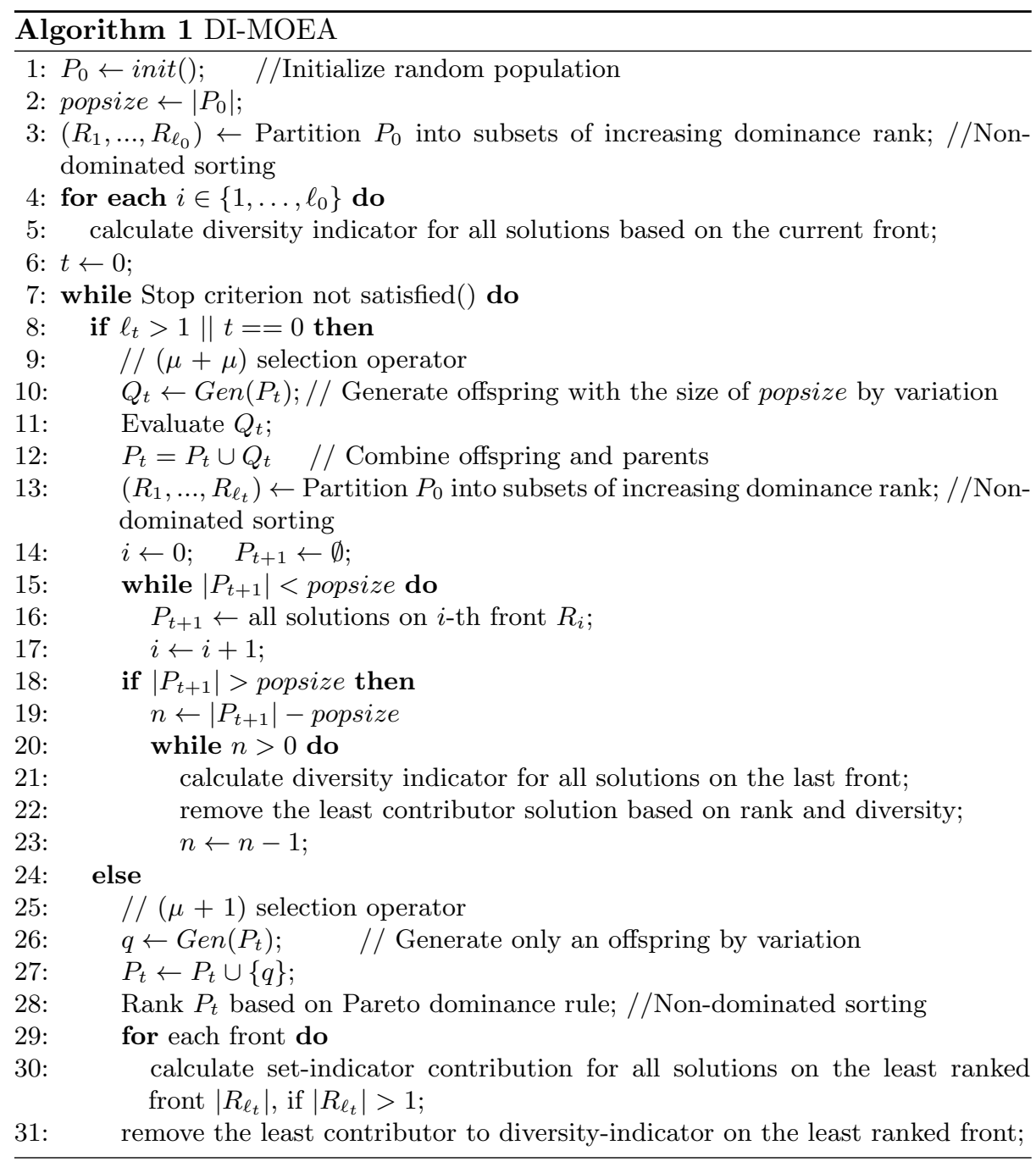

are combined into one. For each problem in the two tables, the upper row denotes the aggregate hypervolume/IGD. (The best value is highlighted in bold.) The lower row is the standard deviation (Std) of results from 30 runs. The MannWhitney $U$ test is used to determine if the medians of different algorithms for the same problem are significantly indifferent. In the tables, we also highlight algorithms whose median performance is indifferent to the algorithm with the best aggregate performance. It can be observed that SMS-EMOA or NSGA-III can achieve the best hypervolume and the best IGD on all three problems, and the proposed DI-MOEA can obtain better hypervolume and IGD than NSGA-II and MOEA/D. In some instances, DI-MOEA can even get better hypervolume and IGD than NSGA-III or SMS-EMOA. 
Table 1. The Aggregate Hypervolume on Bi-Objective Problems

\begin{tabular}{|c|c|c|c|c|c|c|}
\hline Hypervolume & \multirow{2}{*}{ NSGA-II } & \multirow{2}{*}{ SMS-EMOA } & \multirow{2}{*}{ NSGA-III } & \multirow{2}{*}{ MOEA/D } & \multirow{2}{*}{ DI-1 } & \multirow{2}{*}{ DI-2 } \\
\hline Std & & & & & & \\
\hline \multirow{2}{*}{ ZDT1 } & 0.66399 & 0.66602 & 0.66428 & 0.66029 & 0.66473 & 0.66491 \\
\hline & $4.8379 \mathrm{e}-04$ & $7.2331 \mathrm{e}-05$ & $3.9507 \mathrm{e}-04$ & 0.0028 & $3.5973 \mathrm{e}-04$ & $2.8447 \mathrm{e}-04$ \\
\hline \multirow{2}{*}{ ZDT2 } & 0.33002 & 0.33265 & 0.33266 & 0.32849 & 0.33073 & 0.33141 \\
\hline & $4.7756 \mathrm{e}-04$ & $8.7207 \mathrm{e}-05$ & 0.0086 & 0.0030 & $4.9232 \mathrm{e}-04$ & $5.8483 \mathrm{e}-04$ \\
\hline \multirow{2}{*}{ ZDT3 } & 0.51600 & 0.51718 & 0.51720 & 0.51582 & 0.51623 & 0.51634 \\
\hline & $3.9954 \mathrm{e}-04$ & 0.0013 & 0.0010 & 0.0011 & $4.1969 \mathrm{e}-04$ & $2.7955 \mathrm{e}-04$ \\
\hline
\end{tabular}

Table 2. The Aggregate IGD on Bi-Objective Problems

\begin{tabular}{|c|c|c|c|c|c|c|}
\hline \begin{tabular}{|l} 
IGD \\
Std
\end{tabular} & NSGA-II & SMS-EMOA & NSGA-III & MOEA/D & DI-1 & DI-2 \\
\hline \multirow{2}{*}{ ZDT1 } & 0.00163 & 0.00039 & 0.00168 & 0.00385 & 0.00116 & 0.00106 \\
\hline & $2.6517 \mathrm{e}-04$ & $1.9915 \mathrm{e}-05$ & $8.2835 \mathrm{e}-04$ & 0.0018 & $1.4110 \mathrm{e}-04$ & $9.7026 \mathrm{e}-05$ \\
\hline \multirow{2}{*}{ ZDT2 } & 0.00202 & 0.00084 & 0.00051 & 0.00247 & 0.00159 & 0.00120 \\
\hline & $2.1844 \mathrm{e}-04$ & $1.0340 \mathrm{e}-04$ & 0.0088 & 0.0014 & $2.1557 \mathrm{e}-04$ & $2.4062 \mathrm{e}-04$ \\
\hline \multirow{2}{*}{ ZDT3 } & 0.00092 & 0.00037 & 0.00054 & 0.00190 & 0.00087 & 0.00092 \\
\hline & $1.5809 \mathrm{e}-04$ & 0.0100 & 0.0080 & $8.6720 \mathrm{e}-04$ & $1.6713 \mathrm{e}-04$ & $1.3157 \mathrm{e}-04$ \\
\hline
\end{tabular}

\subsection{Experiments on three objective problems}

For three objective problems, DTLZ1 with 7 variables, DTLZ2 with 12 variables and DTLZ7 with 22 variables are tested. Both DI-1 and DI-2 behave very well, and they are indifferent on the the statistical significance of median hypervolume and IGD. Statistical data averaging 10 runs per problem and algorithm are shown on Table 3 and Table 4 . DI- 1 beats all the algorithms on the aggregate hypervolume on all problems, and DI-2 also behaves better than other algorithms except for SMS-EMOA on DTLZ1. For IGD, the new algorithms perform the best on DTLZ1 and DTLZ2 problems. NSGA-II obtains the best IGD on DTLZ7, while IGD values of DI-1 and DI-2 are only slightly lower than NSGA-II on DTLZ7, but better than all other algorithms.

Table 3. The Aggregate Hypervolume on Three Objective Problems

\begin{tabular}{|c|c|c|c|c|c|c|}
\hline Hypervolume & \multirow{2}{*}{ NSGA-II } & SMS-EMOA & NSGA-III & MOEA/D & DI-1 & DI-2 \\
\hline Std $\searrow$ & & & & & & \\
\hline \multirow{2}{*}{ DTLZ1 } & 0.80605 & 0.80732 & 0.78400 & 0.80198 & $\mathbf{0 . 8 0 8 0 6}$ & $\mathbf{0 . 8 0 6 4 5}$ \\
\cline { 2 - 7 } & 0.0062 & $1.8738 \mathrm{e}-04$ & 0.0179 & 0.0015 & 0.0013 & $6.1716 \mathrm{e}-04$ \\
\hline \multirow{2}{*}{ DTLZ2 } & 0.44263 & 0.45269 & 0.41915 & 0.42907 & $\mathbf{0 . 4 5 5 1 1}$ & $\mathbf{0 . 4 5 4 8 9}$ \\
\cline { 2 - 7 } & 0.0070 & $5.8698 \mathrm{e}-05$ & $5.1471 \mathrm{e}-04$ & 0.0031 & 0.0033 & 0.0014 \\
\hline \multirow{2}{*}{ DTLZ7 } & 0.31064 & 0.24694 & 0.30624 & 0.30164 & $\mathbf{0 . 3 1 2 2 7}$ & $\mathbf{0 . 3 1 3 3 9}$ \\
\cline { 2 - 7 } & 0.0034 & 0.0038 & 0.0328 & 0.0055 & 0.0051 & 0.0137 \\
\hline
\end{tabular}


Yali Wang et al.

Table 4. The Aggregate IGD on Three Objective Problems

\begin{tabular}{|c|c|c|c|c|c|c|}
\hline IGD & \multirow{2}{*}{ NSGA-II } & SMS-EMOA & NSGA-III & MOEA/D & DI-1 & DI-2 \\
\cline { 1 - 7 } Std $\searrow$ & & & & & \\
\hline \multirow{2}{*}{ DTLZ1 } & 0.02149 & 0.02074 & 0.04266 & 0.02779 & $\mathbf{0 . 0 1 9 6 6}$ & $\mathbf{0 . 0 2 3 8 1}$ \\
\cline { 2 - 7 } & 0.0063 & $8.1450 \mathrm{e}-04$ & 0.0159 & 0.0018 & 0.0017 & 0.0016 \\
\hline \multirow{2}{*}{ DTLZ2 } & 0.02414 & 0.03415 & 0.05181 & 0.03902 & $\mathbf{0 . 0 1 7 9 9}$ & $\mathbf{0 . 0 1 9 0 9}$ \\
\cline { 2 - 7 } & 0.0047 & 0.0014 & $2.1056 \mathrm{e}-04$ & 0.0026 & 0.0019 & 0.0030 \\
\hline \multirow{2}{*}{ DTLZ7 } & $\mathbf{0 . 0 1 8 2 0}$ & 0.09182 & 0.02381 & 0.041367 & 0.01826 & 0.02191 \\
\cline { 2 - 7 } & 0.0027 & 0.0020 & 0.2151 & 0.0867 & 0.0017 & 0.0944 \\
\hline
\end{tabular}

To easily observe the results of algorithms, we visualized the results on the three objective problems. Figure 1 shows the Pareto front approximations of a typical run on DTLZ1. It can be observed that the solutions of NSGA-II and MOEA/D are not uniformly distributed, and there are several overlaps in the result of NSGA-III. While, SMS-EMOA and our algorithms can obtain evenly spaced solutions on the linear Pareto front.

Figure 2 shows the Pareto front approximations of a typical run on DTLZ2. For NSGA-III, we observed the same phenomenon: some solutions are overlapping or very close. The result of SMS-EMOA is distributed across the Pareto front with emphasis on the boundary and knee regions of the Pareto front. The results of the two DI-MOEA variants are uniformly distributed and evenly spaced on the Pareto front.

DI-MOEA also behaves well on the multimodal DTLZ7 problem, which has non-linear disconnected Pareto front regions. Figure 3 shows the results under 200 population size and $500000 \mathrm{NE}$.

When running the DI-MOEA, it can be observed that the population evolves towards the Pareto front at the initial stage (the first phase) using the generational selection operator. After a short period where the two selection operators alternate (the second phase), the steady state selection operator takes over and the population converges to a set with maximum diversity (the third phase). When the number of objectives becomes large, the third phase is more prominent than the previous two phases because it is more likely for solutions to be mutually non-dominated for a large objective number. In the runs conducted on three objective problems in this paper, the generational selection operator was applied around 100-200 iterations before it switched to the steady state selection operator for the first time. The intermittent alternating phase took about 20-50 iterations, and in most of the running time, the algorithm used the steady state selection operator and throughout this phase, only occasionally the algorithm switched back to generational selection operator for at most a single iteration. Overall, the first and the second phase took only a minor amount of the total running time.

It is worth noting that we observed Dominance Resistant Solutions (DRSs) [8] occasionally on the linear Pareto front of DI-2 on DTLZ1 three objective problem; these are points that have a large contribution to diversity, but domi- 

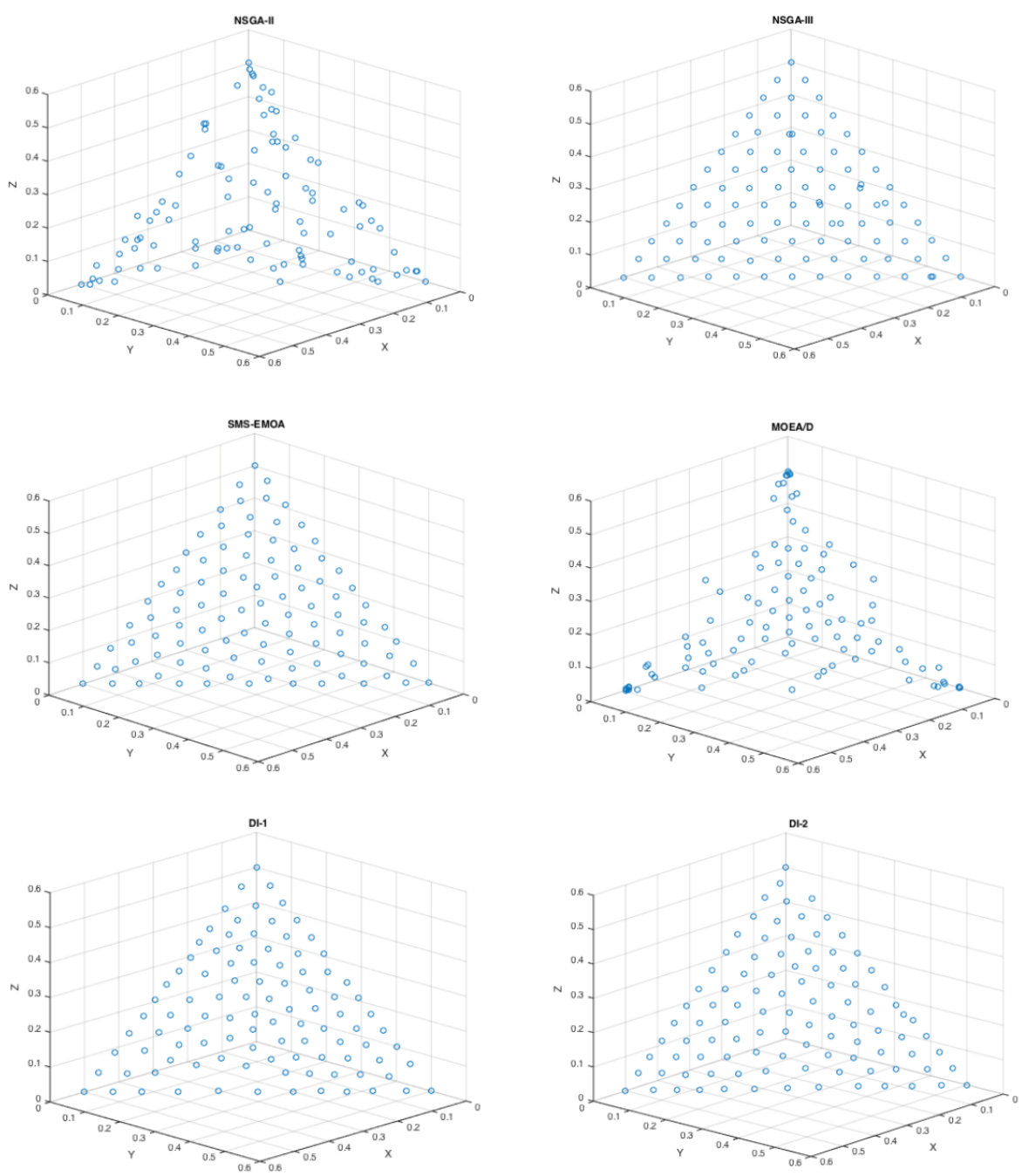

Fig. 1. Representative PF approximations on DTLZ1.

nate only a very narrow region exclusively. It might be necessary to keep these "special solutions", but on the other side, they make the Pareto front approximation less evenly distributed. We already tested a strategy to eliminate DRSs. Before the calculation of the set-indicator contribution for a front, each solution is checked by comparing with all other solutions: the distances between two solutions in all dimensions are calculated, if the result of the minimal distance divided by the maximal distance is too small, the current solution will be removed from the front. Therefore, a shrinked front is created and diversity indicator can be calculated only in the new front. The underlying idea of this strategy is that 
Yali Wang et al.
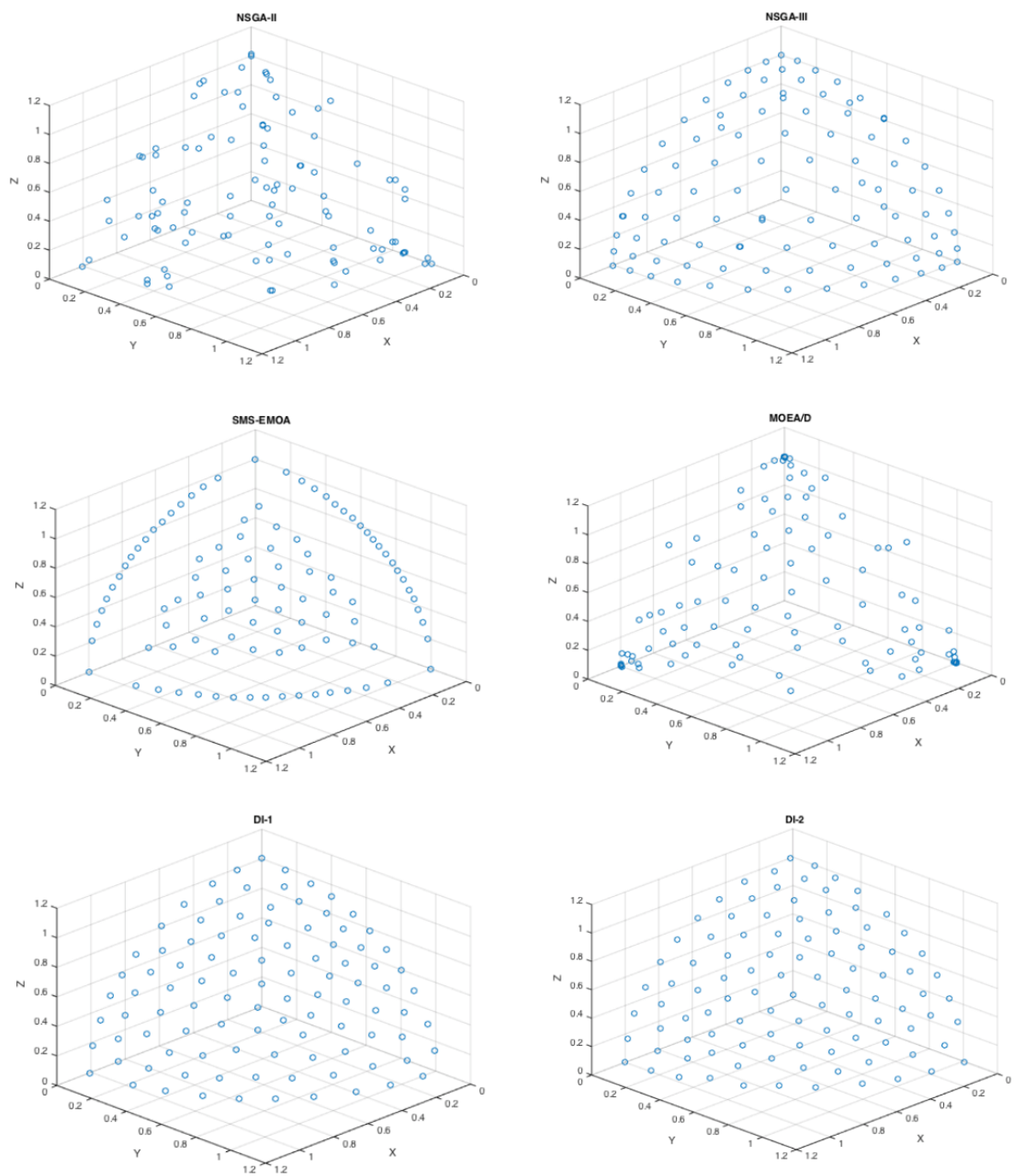

Fig. 2. Representative PF approximations on DTLZ2.

for two solutions, if their distance is too close in one dimension and too large in another dimension, keeping both of them will result in an uneven distribution.

\section{Conclusions and Further Work}

The proposed DI-MOEA combines the advantage of Pareto dominance-based and indicator-based methods. Moreover, the achieved Pareto front approximations are excellent in both hypervolume indicator and IGD. Especially, the rel- 

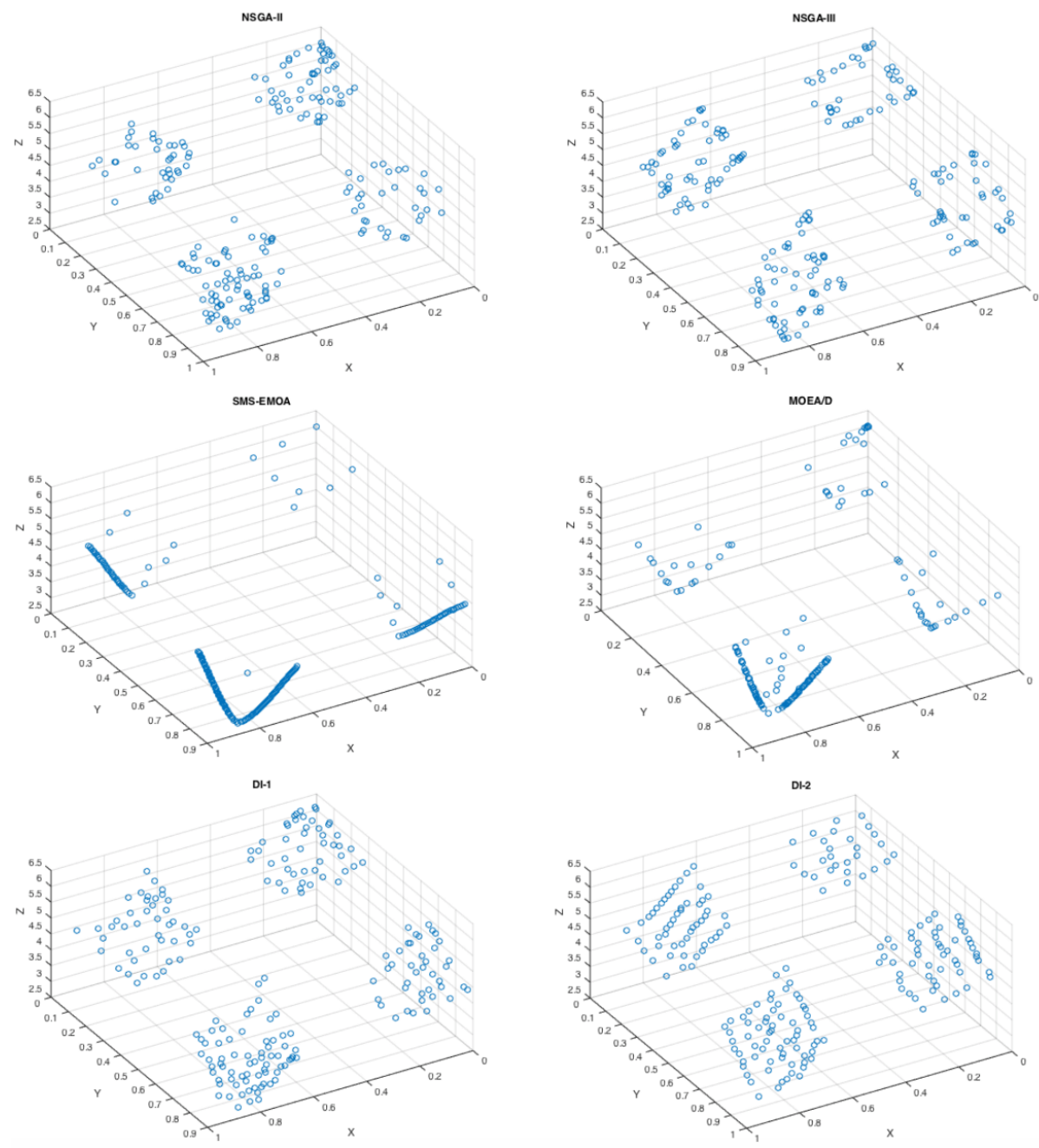

Fig. 3. Representative PF approximations on DTLZ7.

ative performance of our algorithms even gets better with increasing number of objectives. The set-indicator used in our algorithms is computationally simpler than the hypervolume indicator and only depend linearly on the number of objectives, making it possesses a potential advantage on MaOPs. Most importantly, the uniformly distributed, evenly spaced solution set can be achieved without the use of decomposition sets and the estimation of the location and shape of the true Pareto front.

In the current implementation of DI-MOEA, only a naive way of calculating the Euclidean distance based geometric mean gap is implemented. Although the computational time of the implemented algorithm is shorter than SMS- 
EMOA, it should be further improved, e.g., by using Vaidya's algorithm [11] and incremental updates of contributions. Besides, the new DI-EMOA holds the promise of performing well in many-objective optimization. To study this, its performance should be tested on MaOP benchmarks, paying special attention to effects that might occur in high dimensional objective spaces, such as distance concentration and the increasing number of non-dominated solutions. Also, more MOEAs can be involved in comparison, such as MACE-gD [6] and IBEA [14].

\section{References}

1. N. Beume, B. Naujoks, and M. Emmerich. SMS-EMOA: Multiobjective selection based on dominated hypervolume. European Journal of Operational Research, 181(3):1653-1669, 2007.

2. K. Deb, S. Agrawal, A. Pratap, and T. Meyarivan. A fast and elitist multiobjective genetic algorithm: NSGA-II. IEEE Trans. Evolutionary Computation, 6(2):182197, 2002.

3. Kalyanmoy Deb and Himanshu Jain. An evolutionary many-objective optimization algorithm using reference-point-based nondominated sorting approach, part i: Solving problems with box constraints. IEEE Trans. Evolutionary Computation, 18(4):577-601, 2014.

4. MTM Emmerich, AH Deutz, and JW Kruisselbrink. On quality indicators for black-box level set approximation. In EVOLVE-A bridge between probability, set oriented numerics and evolutionary computation, pages 157-185. Springer, 2013.

5. Jay B Ghosh. Computational aspects of the maximum diversity problem. Operations research letters, 19(4):175-181, 1996.

6. I. Giagkiozis, R. C. Purshouse, and P. J. Fleming. Generalized decomposition and cross entropy methods for many-objective optimization. Information Sciences, 282:363-387, 2014.

7. P. Hajela and C-Y Lin. Genetic search strategies in multicriterion optimal design. Structural optimization, 4(2):99-107, 1992.

8. Thomas Hanne. On the convergence of multiobjective evolutionary algorithms. European Journal of Operational Research, 117(3):553-564, 1999.

9. L.-Y. Liu, V. Basto-Fernandes, I. Yevseyeva, J. Kok, and M. Emmerich. Indicatorbased evolutionary level set approximation: Mixed mutation strategy and extended analysis. In International Work-Conference on the Interplay Between Natural and Artificial Computation, pages 146-159. Springer, 2017.

10. T. Ulrich, J. Bader, and L. Thiele. Defining and optimizing indicator-based diversity measures in multiobjective search. In International Conference on Parallel Problem Solving from Nature, pages 707-717. Springer, 2010.

11. P. M. Vaidya. An o(n $\log \mathrm{n})$ algorithm for the all-nearest-neighbors problem. Discrete \& Computational Geometry, 4(2):101-115, Mar 1989.

12. S. Wessing. Two-stage methods for multimodal optimization. PhD thesis, Universitätsbibliothek Dortmund, 2015.

13. Q. Zhang and H. Li. Moea/d: A multiobjective evolutionary algorithm based on decomposition. IEEE Transact. on Evol. Comp., 11(6):712-731, 2007.

14. Eckart Zitzler and Simon Künzli. Indicator-based selection in multiobjective search. In International Conference on Parallel Problem Solving from Nature, pages 832-842. Springer, 2004. 